\title{
APPLYING MACHINE LEARNING TECHNIQUES TO DNA SEQUENCE ANALYSIS
}

\author{
Jude W. Shavlik, Principal Investigator \\ Computer Sciences Department \\ University of Wisconsin - Madison \\ shavlik@cs.wisc.edu.(608)262-7784
}

DOE/ER/61129--T1

\section{PROJECT OVERVIEW}

We are developing a machine learning system that modifies existing knowledge about specific types of biological sequences. It does this by considering sample members and nonmembers of the sequence motif being learned. Using this information (which we call a "domain theory"), our learning algorithm produces a more accurate representation of the knowledge needed to categorize future sequences. Specifically, the KBANN algorithm [1] maps inference rules, such as consensus sequences, into a neural (connectionist) network. Neural network training techniques then use the training examples to refine these inference rules. We rave been applying this approach to several problems in DNA sequence analysis and have also been extending the capabilities of our learning system along several dimensions. These activities are summarized below.

\section{SPECIFIC DNA PROBLEMS STUDIED}

\section{E. coli Promoter Recognition}

In collaboration with Prof. F. Blattner of the Wisconsin Genetics Department (whose laboratory is sequencing $E$. coli), we have been creating a neural network recognizer of promoters. We created and extensively analyzed empirically an initial domain theory for promoter recognition. This analysis $[2,3]$ highlighted weaknesses in the initial theory, and based on further review of the literature on prokaryotic promoters, we created a more extensive domain theory. We also increased the number of sample promoters by a factor of five and the number of negative examples (i.e., non-promoters) by a factor of 100 . We increased the capacity of our implementation of the KBANN algorithm and trained a more accurate promoter recognizer. We are using this recognizer to analyze Blattner's $E$. coli sequence data.

\section{Splice Junction Recognition}

We also trained a recognizer of eukaryotic splice junctions. Our initial domain theory for this problem was very sparse, and we have developed algorithmic methods for expanding (rather than simply refining) this domain theory during the training process [4]. The initial results demonstrated that this process can increase accuracy, and we are continuing development along these lines. Over the next year, we plan to make our trained networks publicly accessible.

\section{Detecting Coding Regions and Frameshifts in Prokaryotic DNA Sequences}

More recently, we have been applying our learning methods to the problem of predicting the reading frames in prokaryotic DNA, which is useful for detecting coding regions and frameshift errors. We have collected a large set of training sequences and written some preliminary domain theories. We also implemented programs by Staden and Gribskov for comparative purposes. We have found this task to be harder than distinguishing exons from introns (we only get about $65 \%$ accuracy on the reading-frame problem) and are investigating several extensions to
increase performance. 


\section{DISCLAIMER}

This report was prepared as an account of work sponsored by an agency of the United States Government. Neither the United States Government nor any agency thereof, nor any of their einployees, makes any warranty, express or implied, or assumes any legal liability or responsibility for the accuracy, completeness, or usefulness of any information, apparatus, product, or process disclosed, or represents that its use would not infringe privately owned rights. Reference herein to any specific commercial product, process, or service by trade name, trademark, manufacturer, or otherwise does not necessarily constitute or imply its endorsement, recommendation, or favoring by the United States Government or any agency thereof. The views and opinions of authors expressed herein do not necessarily state or reflect those of the United States Government or any agency thereof. 


\section{Sequence Alignment in the Presence of Frameshift Errors}

In work complementary to our neural network work, we developed an algorithm that performs sequence alignment in the presence of frameshift errors [5]. With the joint support of Blattner's $E$. coli grant, we also created a sophisticated "X windows" interface to this program. This system is used on an everyday basis in Blattner's laboratory to both detect sequencing errors and to perform a preliminary analysis of the sequences they produce. It also provides our group with useful training and benchmarking data (e.g., for promoter and coding-region finding).

\section{New Problems being Initiated}

We have also begun talking to biologists about several other problems. With the assistance of Dr. G. Michaels of NIH, we are considering applying KBANN to the task of recognizing zincfinger binding sites. We are also applying our techniques to the refinement of DNA grammars (a more powerful formalism than we can currently handle in our domain theories); this is in collaboration with Prof. D. Searls of the University of Pennsylvania.

\section{ALGORITHMIC IMPROVEMENTS}

We have created several extensions to our basic algorithms that are applicable to a wide range of specific problems.

\section{Rule Extraction}

One problem with neural networks is that the information they contain is in an opaque form (basically, large arrays of numbers). Hence it is hard to understand what it is that they have learned. To overcome this shortcoming, we have developed a method for extracting "rules" from trained neural networks [2, 3, 6-9]. Our algorithm produces comprehensible rules whose accuracy on the promoter and splice-junction tasks exceeds that of the trained network from which they were extracted. We are highly encouraged by these results and are extending our investigation of methods for rule extraction.

\section{Visualization of Neural Networks}

We have developed a software tool, called the "visualizer", that also aids in the understanding of neural networks [10]. By viewing a neural network pictorially, a better understanding can be gained of how it learns and solves a given problem. This X Windows-based tool allows the user of KBANN to see how his or her domain theory is mapped into a neural network, as well as watch the training process. Also, this tool can be used by biologists, who need know nothing about neural networks, to observe how trained networks classify new examples. For example, we are using this tool to scan $E$. coli sequences for promoters. Currently we are working on making this tool freely available via ftp (i.e., electronic file transfer).

\section{More Focused Training of KBNs}

The essence of our research is the ability to refine domain-specific knowledge on the basis of training examples. Hence, an important goal is to improve the backpropagation-based training process. We are developing extensions to backpropagation that explicitly take advantage of the fact that, unlike most work on neural networks, the topology and semantics of our networks are based on a domain theory. We have found that our extensions improve accuracy [11]. 


\section{MISCELLANEOUS ACTIVITIES}

We placed our domain theories and data sets in the UC-Irvine archive of machine learning problems. This has enabled others in the machine learning community to work on these problems. We know that research groups at the University of Texas - Austin, Johns Hopkins University, Cornell University, University of Illinois - Urbana, University of Florida, and NASA-Ames are using this data (other users may not have informed us).

This grant supported the completion of Geoff Towell's Ph. D. dissertation in computer science. He was offered a research position at the National Library of Medicine, to continue work on the application of machine learning to biological problems. However, he instead accepted a position at Siemens Research Laboratories in New Jersey.

The PI helped organize a NIH-NSF jointly-sponsored meeting on "Creating an Infrastructure for Intelligent Systems in Molecular Biology." He also served as a reviewer on the team that visited Lawrence Berkeley Laboratory's genome center (January 1992).

Portions of this work (specifically, the algorithmic improvements) have been partially supported by grants from NSF, ONR, and IBM.

\section{SUPPORTED PUBLICATIONS}

1. G. G. Towell and J. W. Shavlik, "Knowledge-Based Neural Networks," submitted to Artificial Intelligence.

2. J. W. Shavlik, G. G. Towell and M. O. Noordewier, "Using Neural Networks to Refine Biological Knowledge," International Journal of Genome Research 1, 1 (in press).

3. G. G. Towell, "Symbolic Knowledge and Neural Networks: Insertion, Refinement, and Extraction," Ph.D. Thesis, Department of Computer Science, University of Wisconsin, Madison, WI, Dec 1991.

4. G. G. Towell, M. W. Craven and J. W. Shavlik, "Constructive Induction in Knowledge-Based Neural Networks," Proceedings of the Eighth International Conference on Machine Learning, Evanston, IL, Junc 1991, pp. 213-217.

5. J. W. Shavlik, "Finding Genes by Case-Based Reasoning in the Presence of Noisy Case Boundaries," Proceedings of the DARPA Cased-Based Reasoning Workshop, May 1991, pp. 327-338.

6. G. G. Towell and J. W. Shavlik, "Refining Symbolic Knowledge Using Neural Networks," in Machine Learning: An Integrated Approach, Vol.IV, R. S. Michalski and G. Tecuci (cds.), Morgan Kaufmann, San Mateo, CA, in press.

7. G. G. Towell and J. W. Shavlik, "The Extraction of Refined Rules from Knowledge-Based Neural Networks," submitted to Machine Learning.

8. G. G. Towell and J. W. Shavlik, "Interpretation of Artificial Neural Networks: Mapping Knowledge-Based Neural Networks into Rules," in Advances in Neural Information Processing Systems, R. Lippmann, J. Moody and D. Tourctzky (eds.), Morgan-Kaufmann, Los Altos, CA, 1992.

9. G. G. Towell and J. W. Shavlik, "Refining Symbolic Knowledge Using Neural Networks," Proceedings of the International Workshop on Multistrategy Learning, Harper's Ferry, WV, November 1991.

10. M. W. Craven and J. W. Shavlik, "Visualizing Learning and Computation in Neural Networks," International Journal on Artificial Intelligence Tools 1, 2 (in press).

11. G. G. Towell and J. W. Shavlik, "Using Symbolic Inductive Learning to Improve Knowledge-Based Neural Networks," submitted to the National Conference on Artificial Intelligence, 1992. 


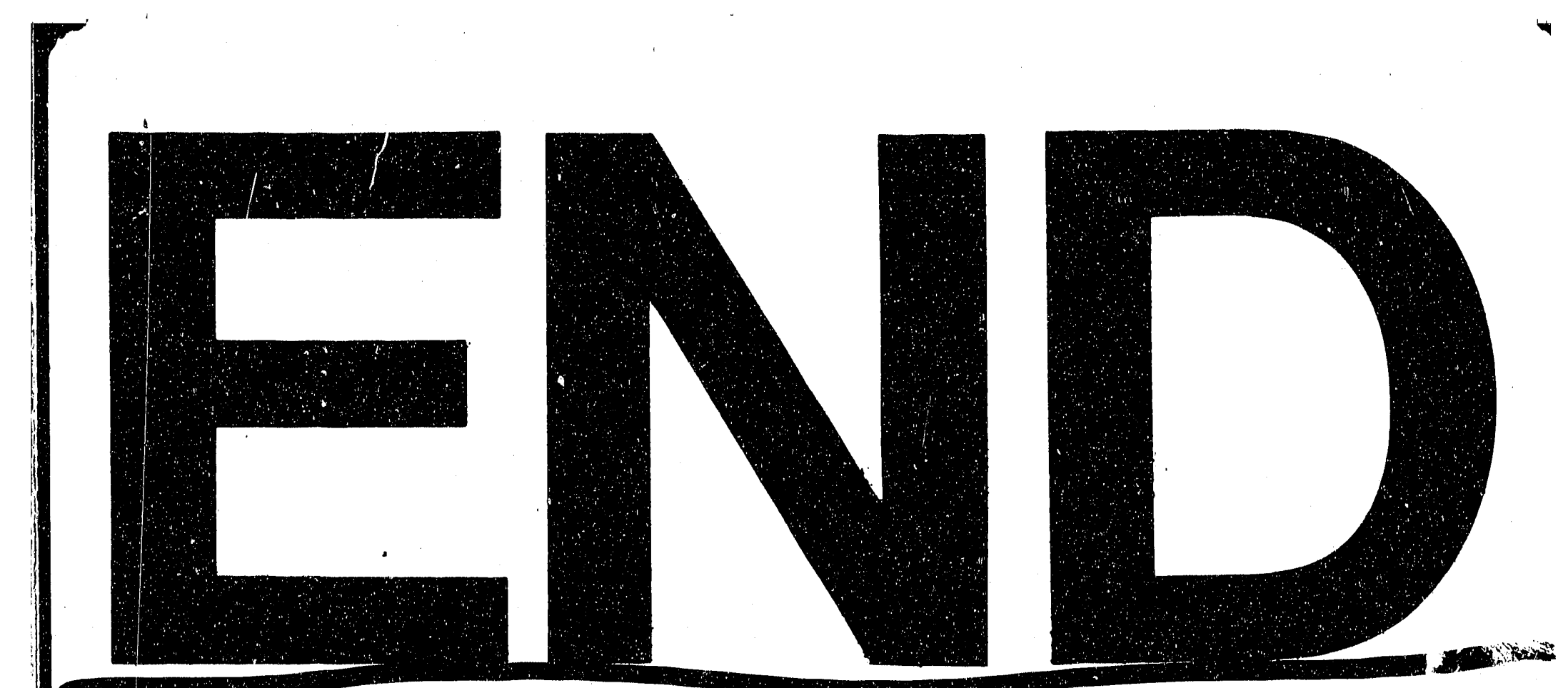




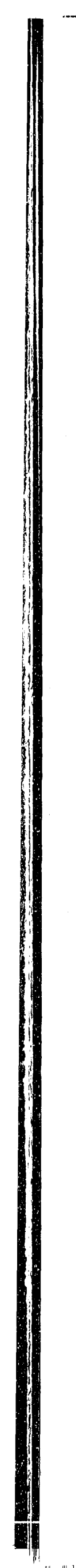

